\title{
Estrogen promotes fat mass and obesity-associated protein nuclear localization and enhances endometrial cancer cell proliferation via the mTOR signaling pathway
}

\author{
YAPING ZHU, JIAQI SHEN, LIYAN GAO and YOUJI FENG \\ Department of Obstetrics and Gynecology, Shanghai Jiao Tong University \\ Affiliated First People's Hospital, Shanghai 200080, P.R. China \\ Received October 15, 2015; Accepted January 11, 2016
}

DOI: $10.3892 /$ or.2016.4613

\begin{abstract}
Extensive exposure to estrogen is generally acknowledged as a risk factor for endometrial cancer. Given that the accumulation of adipocytes also contributes to the increased production of estrogen, in the present study, we evaluated the expression of the fat mass and obesity-associated (FTO) gene in endometrial tumor tissues and further explored the mechanism of how estrogen facilitates FTO nuclear localization and promotes endometrial cancer cell proliferation. Immunohistochemical (IHC) staining assay was used to detect the FTO expression in endometrial tumor samples. Western blotting was performed to investigate the mechanism of estrogen-induced FTO nuclear localization. siRNA was used to knock down ER $\alpha$ and further explore its role in FTO nuclear localization. MTT assay was carried out to determine cell proliferation. We found that FTO was overexpressed in endometrial carcinoma tissues and served as a poor prognostic marker. Additionally, estrogen induced FTO nuclear accumulation via the mTOR signaling pathway and the nuclear localization was ER $\alpha$-dependent, which contributed to enhanced proliferative activity. Therefore, the present study provides new insight into the mechanisms of estrogen-induced proliferation, implying the possibility of using FTO as a potential therapeutic target for the treatment of endometrial cancer.
\end{abstract}

\section{Introduction}

Endometrial cancer is one of the most common gynecologic malignancies worldwide $(1,2)$. There are two different clinicopathological types of endometrial carcinoma, including type I and II endometrial carcinoma (3). In clinical practice,

Correspondence to: Professor Yaping Zhu or Professor Youji Feng, Department of Obstetrics and Gynecology, Shanghai Jiao Tong University Affiliated First People's Hospital, 100 Haining Road, Shanghai 200080, P.R. China

E-mail: zhuyaping2008@163.com

E-mail: fengyj4806@sohu.com

Key words: estrogen, endometrial cancer, FTO, nuclear localization over $80 \%$ of endometrial cancers are type I, which commonly comprise low-grade carcinomas. These tumors are often preceded by endometrial premalignant disease and are always estrogen nuclear receptor (ER) and progesterone receptor (PR)-positive, which frequently arise during excessive estrogen exposure. Recently, much effort has been made to confirm the involvement of aberrant estrogen metabolism in dysregulated endometrial cancer cell growth and malignant metastasis (4-6). We previously suggested that estrogen promoted endometrial cancer cell proliferation and invasion by fat mass and obesityassociated (FTO) gene (7), however, the molecular mechanism of how FTO regulates cellular growth by estrogen remains obscure.

FTO was identified as an oncoprotein frequently overexpressed in several types of cancer, including endometrial, breast and pancreatic cancer (8-13). FTO is a protein involved in energy homeostasis by controlling energy expenditure. Depletion of FTO in mice was found to result in growth retardation, adipose tissue reduction and lean body mass (14). Since estrogen-driven endometrial cancer is strongly and definitively linked to obesity, two studies previously examined the relationship between FTO gene polymorphism and the incidence of endometrial cancer $(9,13)$. In our previous study, we also found that estrogen stimulation resulted in FTO accumulation in the nucleus (7), however, the mechanism of estrogen-driven FTO nuclear localization is not clear.

Although less than $0.1 \%$ of the total cellular protein, kinase and phosphatase enzymes play a pivotal role in conducting signals to control cell growth or invasion, abundant signaling pathways have been reported to be involved in estrogendriven endometrial cancer. Inhibition of the PI3K/AKT pathway was found to lead to a decrease in proliferative and invasive activities. Blocking MAPK signaling also resulted in similar effects. These results are consistent with our previous study (7). Another important signaling molecule, mTOR is an atypical serine/threonine protein kinase that belongs to the phosphoinositide 3-kinase (PI3K)-related kinase family and is involved in energy metabolism $(15,16)$. mTOR has been implicated in the development and progression of various types of cancers including melanoma, lung and endometrial cancer (17-27). Recent findings suggest that the mTOR pathway may play an important role in endometrial cancer 
progestin resistance (28). However, as a molecule controlling energy expenditure, whether estrogen-driven FTO nuclear localization is mediated by the mTOR signaling pathway has not been studied. Therefore, the aim of the present study was to investigate whether estrogen enhances FTO nuclear localization and promotes endometrial cancer cell growth via the mTOR signaling pathway.

\section{Materials and methods}

Sample collection. Forty-nine samples were obtained from the Tissue Bank of the Department of Obstetrics and Gynecology of the Shanghai First People's Hospital affiliated to Shanghai Jiao Tong University, which were comprised of 18 normal endometrial tissues and 31 cases of type I endometrial carcinoma. None of the patients in the study had a history of prior radiotherapy or chemotherapy. Any patient with a known history of hormone replacement was excluded. The use of these specimens was approved by the Ethics Committee of the Medical College of Shanghai Jiao Tong University, China.

Immunohistochemical (IHC) staining and analysis. IHC analysis of FTO protein expression was performed as previously described and assessed using a semi-quantitative method. Briefly, specimens were deparaffinized in xylene and rehydrated in a graded series of ethanol and subsequently endogenous peroxidase activity was blocked by a 10 -min treatment with $3.0 \%$ hydrogen peroxide. Subsequently, the sections were subjected to antigen retrieval by boiling in citrate buffer (pH 6.0) and incubated for 30 min with $0.01 \%$ Trixon and then incubated for $20 \mathrm{~min}$ with $5 \%$ bovine serum albumin (BSA). The sections were incubated overnight with a rabbit antihuman FTO primary antibody at $4^{\circ} \mathrm{C}$ in a humidity chamber, followed by a 50-min incubation with a biotinylated secondary antibody (Dako, Carpinteria, CA, USA). Omitted primary antibodies served as negative controls. Expression of FTO protein was assessed using a semi-quantitative method: the slides were evaluated for the percentage of positively stained cells (0-4) and the intensity of the staining (0-3). The index of FTO expression was calculated as the percentage $\mathrm{x}$ intensity of the staining. Therefore, a score of 0 is negative (-), 1-4 is weakly positive $(+), 5-8$ is positive $(++)$, and $9-12$ is strongly positive $(+++)$.

Cell lines and cell culture. To investigate the mechanism of estrogen-induced FTO nuclear localization, the Ishikawa cell line was used in the present study, which is an estrogen-responsive cell line derived from a well-differentiated endometrioid carcinoma. The cells were maintained in our laboratory after being generously provided by Dr Masato Nishida, Tsukuba University, Tsukuba City, Japan. The cells were maintained in Dulbecco's modified Eagle's medium (DMEM)/F-12 (1:1) medium with $10 \%$ fetal bovine serum (FBS) (both from Gibco, Gaithersburg, MD, USA), $100 \mathrm{U} / \mathrm{ml}$ penicillin, sodium pyruvate and L-glutamine in a humidified atmosphere of $5 \%$ $\mathrm{CO}_{2}$ at $37^{\circ} \mathrm{C}$.

Immunoblot analysis. Immunblot analysis was performed as previously described. Briefly, the harvested cells were lysed and the supernatant was collected. Then, the protein was loaded onto SDS-PAGE and transferred to polyvinylidene fluoride (PVDF) membrane. The membranes were blocked with $5 \%$ skimmed milk for $1 \mathrm{~h}$ and incubated overnight with the primary antibodies, followed by $1 \mathrm{~h}$ of incubation with the appropriate secondary antibody $(1: 5,000)$. The anti-GAPDH or anti-lamin B1 rabbit monoclonal antibody was diluted to 1:1,000 for use as a sample loading control. The antibodies for FTO, GAPDH, lamin B1, p-mTOR, mTOR and ER $\alpha$ were purchased from Abcam (Cambridge, UK).

Subcellular fractionation. Ishikawa cells treated with estrogen, rapamycin or transfected with $\operatorname{siER} \alpha$ were harvested and lysed with cytoplasmic extraction buffer (10 mM HEPES $\mathrm{pH}$ 7.9, $10 \mathrm{mM} \mathrm{KCl,} 0.1 \mathrm{mM}$ EDTA, $1.5 \mathrm{mM} \mathrm{MgCl}, 1 \mathrm{mM}$ dithiothreitol, $0.2 \%$ Nonidet P-40, $1 \mathrm{mM} \mathrm{NaF}, 1 \mathrm{mM} \mathrm{Na} \mathrm{VO}_{4}$ and protease inhibitor cocktail). After being centrifuged at $14,000 \mathrm{rpm}$ at $4^{\circ} \mathrm{C}$ for $5 \mathrm{~min}$, the cytoplasmic fraction was collected. Then, the pellet was re-suspended in nuclear extraction buffer (20 mM HEPES pH 7.9, $420 \mathrm{mM} \mathrm{NaCl}$, $0.1 \mathrm{mM}$ EDTA pH 8.0, $1.5 \mathrm{mM} \mathrm{MgCl}_{2}, 1 \mathrm{mM}$ dithiothreitol, $0.2 \%$ Triton-X 100, $1 \mathrm{mM} \mathrm{NaF}, 1 \mathrm{mM} \mathrm{Na} \mathrm{VO}_{4}$ and protease inhibitor cocktail), and the nuclear fraction was collected after a 5-min centrifugation.

Immunocytochemistry. Ishikawa cells treated with $10^{-9} \mathrm{M}$ E2 for $48 \mathrm{~h}$ were cultured on coverslips before fixation with $3.7 \%$ paraformaldehyde in phosphate-buffered saline (PBS) (10 min), permeabilization with $0.2 \%$ Triton X-100 (10 min), blocking in $3 \%$ BSA $(1 \mathrm{~h})$ and then incubation with an anti-human FTO primary antibody (1:100, overnight). After incubation with a FITC-labeled secondary antibody (1 h at room temperature), the cells were photographed.

Small interfering RNA (siRNA) transfection and hormone stimulation. The acute knockdown of $\operatorname{siER} \alpha$ was performed as previously described. Briefly, Ishikawa cells were seeded in $5 \mathrm{ml}$ of growth medium in 6-cm dishes without antibiotics, and grown to $30-50 \%$ confluency $24 \mathrm{~h}$ prior to transfection with 200 pmol ER $\alpha$ siRNA (Shanghai GenePharma Co., Ltd.) using DharmaFECT (Thermo Scientific). siRNA-treated and untreated Ishikawa cells were exposed to $1 \mathrm{nM}$ E2 for a further $48 \mathrm{~h}$ before being collected for western blot analysis. The FTO knockdown was performed in a 96-well with the incubated Ishikawa cells, and the cell proliferation was determined by MTT assay.

MTT assay. To investigate the proliferative activity of endometrial cancer cells after various treatments, the MTT assay was performed. Briefly, Ishikawa cells were plated in a 96-well plate $(2,000$ cells/well) and incubated for $24 \mathrm{~h}$. The culture medium was then changed to serum-free DMEM/F-12 (1:1) medium for $24 \mathrm{~h}$. In order to determine the effect of ICI or Rap on cellular growth, cells were pre-treated with ICI or Rap for $1 \mathrm{~h}$ after stimulation with $1 \mathrm{nM}$ E2 or dimethyl sulfoxide (DMSO) for $48 \mathrm{~h}$. Similarly, prior to $1 \mathrm{nM}$ E2 stimulation, the cells were transiently transfected with FTO siRNA to investigate its role in cell proliferation. MTT solution $(20 \mu \mathrm{l}$ of $5 \mathrm{mg} / \mathrm{ml} \mathrm{MTT}$ in PBS) was added to the cells. After $4 \mathrm{~h}$ of incubation at $37^{\circ} \mathrm{C}$, the culture medium was removed, and $150 \mu \mathrm{l}$ of DMSO was added to dissolve the formazan. Finally, 
A
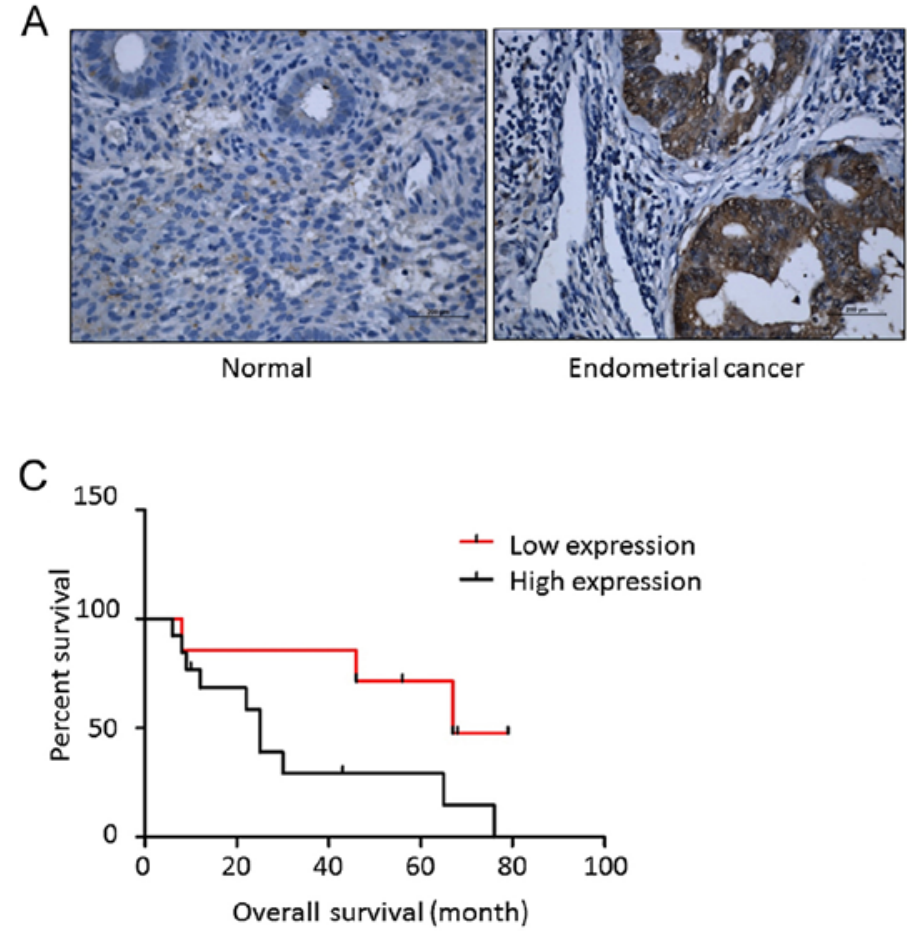

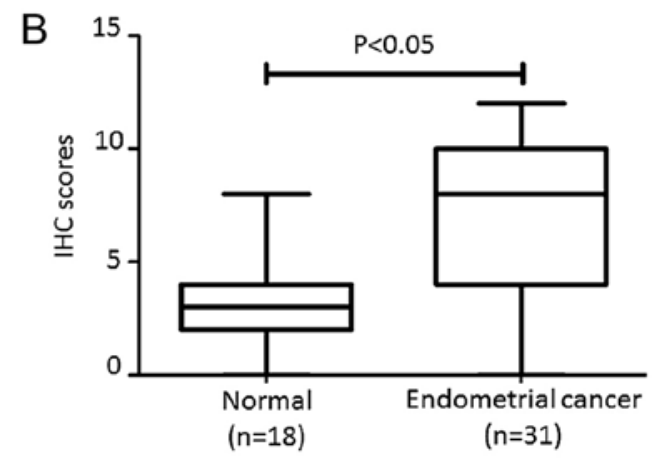

D

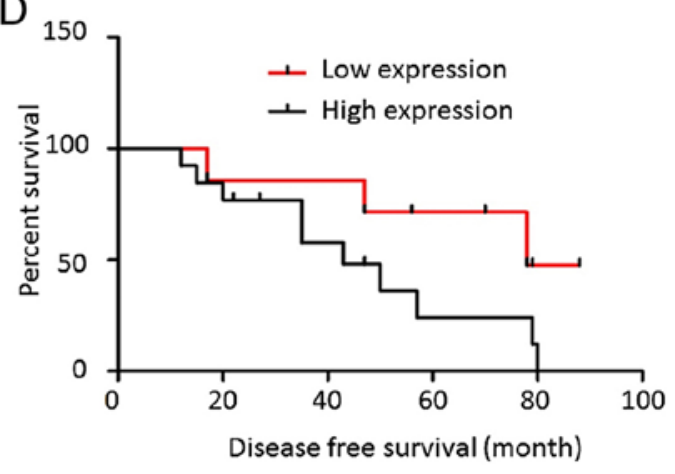

Figure 1. Type I endometrial cancer exhibits elevated FTO expression. (A) The expression levels of FTO in normal endometrial tissues and cancers were assessed using IHC staining. (B) FTO IHC scores in normal endometrial samples and cancers. (C and D) The overall survival and disease-free survival curves of endometrial cancer patients with different expression of FTO.
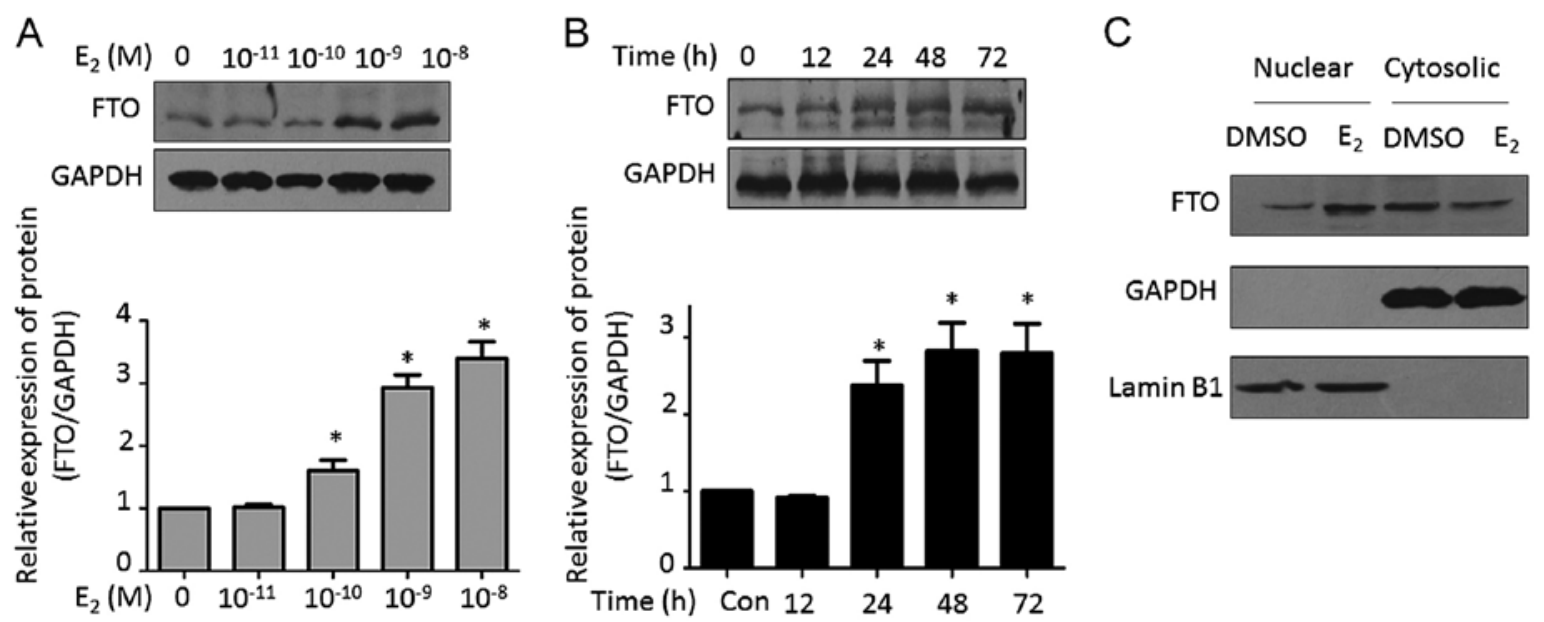

Figure 2. Estrogen promotes FTO nuclear localization. Western blotting was used to determine the FTO expression pattern after treatment with different doses of estrogen (A) and for different times (B). The upper panels are representative bands of the western blotting. Lower panels show statistical analysis of changes in FTO protein after the above indicated treatments. ${ }^{*}<0.05$, compared with the control. (C) After treatment with estrogen for $48 \mathrm{~h}$, western blotting was used to detect the alteration of FTO expression in the nuclear and cytosolic fractions. GAPDH and lamin B1 served as loading controls. Ishikawa cells were used in the present study.

absorbance at $490 \mathrm{~nm}$ was measured with a GENios multifunction reader (Tecan, Zurich, Switzerland).

Statistical analysis. The statistical significance of the differences in the IHC staining in endometrial tissues was calculated using the Chi-square test. The differences in various protein levels and cell proliferation between groups were analyzed using the Student's t-test. A two-sided test with $\mathrm{P}<0.05$ was considered statistically significant. All statistical analyses were performed using SPSS 11.0 (SPSS, Inc., Chicago, IL, USA).

\section{Results}

FTO is overexpressed in endometrial cancer and serves as a marker for poor prognosis. To understand the role of FTO in endometrial cancer development, we first examined the expression of FTO in endometrial carcinoma tissues. As shown in Fig. 1A, little positive staining was detected in the normal endometrial tissues while strong positive staining was observed in type I endometrial carcinomas. The significant difference is summarized in Fig. 1B. Although we reported 
A

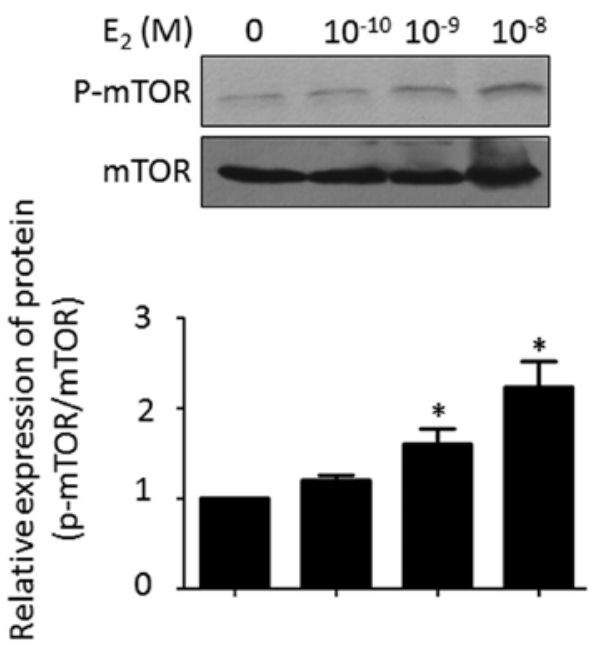

C
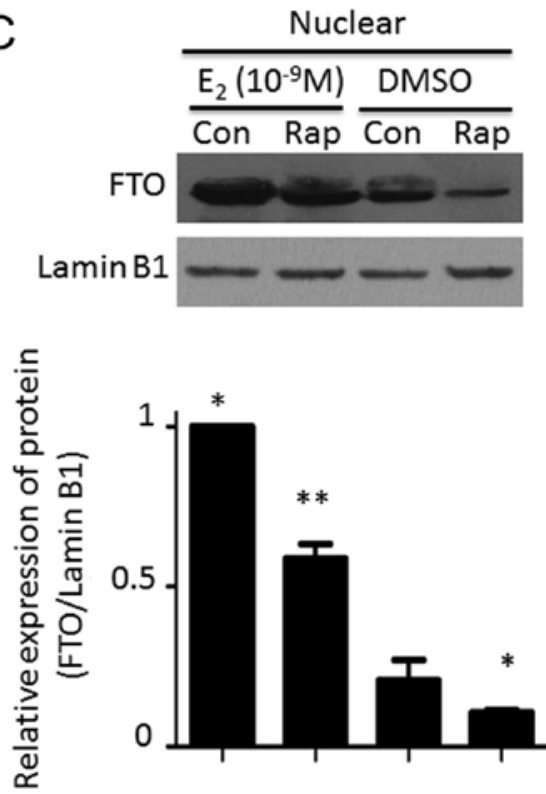

B
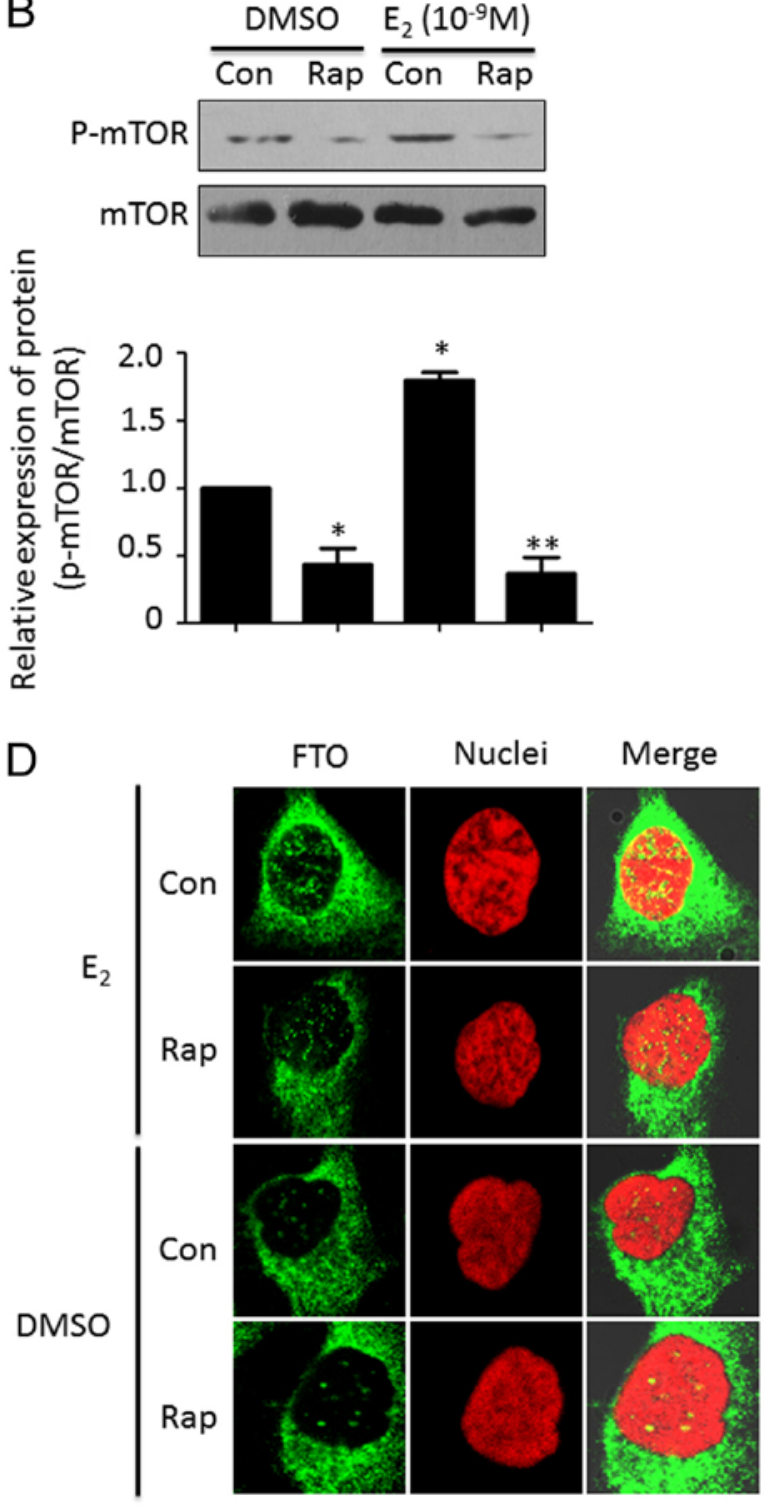

Figure 3. Estrogen controls FTO nuclear localization through the mTOR signaling pathway. (A) Activation of mTOR signaling was determined after estrogen treatment at different doses. The upper panels are representative bands of the western blotting. Lower panels show statistical analysis of changes in mTOR signaling (p-mTOR/mTOR) after treatment with different dose of estrogen. ${ }^{*} \mathrm{P}<0.05$, compared with the control. (B) Effects of rapamycin (Rap) (100 nM) on estrogen-induced $\left(10^{-9} \mathrm{M}\right)$ activation of mTOR signaling. Effects of rapamycin $(100 \mathrm{nM})$ on estrogen-induced (10-9 M) FTO nuclear localization were evaluated by western blotting (C) and immunocytochemical assay (D).

that FTO expression was not associated with age, stage, grade, invasion and lymph node metastasis, we still found that higher FTO expression correlated with poor prognosis and early relapse (Fig. 1C and D).

Estrogen promotes FTO nuclear localization. In our previous study, increasing nuclear expression of FTO was observed after estrogen stimulation in endometrial cancer cells (7). Consistent with this result, we found that estrogen promoted FTO expression in a dose-dependent manner. The major stimulating peak was observed with 1-10 $\mathrm{nM}$ estrogen treatment (Fig. 2A). Moreover, estrogen also induced FTO expression in a time-dependent manner. After treatment with $1 \mathrm{nM}$ estrogen for $48 \mathrm{~h}$, a significant stimulating effect was observed (Fig. 2B). We further isolated the nuclear and cytosolic proteins. Estrogen-induced FTO was detected, however, there was no significant alteration of FTO expression in the cytosol following estrogen treatment (Fig. 2C).

Estrogen controls FTO nuclear localization through the mTOR signaling pathway. To understand the mechanism of estrogen-induced FTO nuclear localization, we scanned the signaling pathways which may be involve in this event. As shown in Fig. 3A, estrogen increased the phosphorylation of mTOR in a dose-dependent manner. Pretreatment with rapamycin potently blocked the estrogen-activated mTOR signaling pathway (Fig. 3B). After combined treatment with estrogen and rapamycin, we found that estrogen-induced FTO nuclear localization was attenuated. The FTO expression pattern in the nucleus following estrogen plus rapamycin treatment was lower than that with estrogen stimulation alone (Fig. 3C and D). 
A

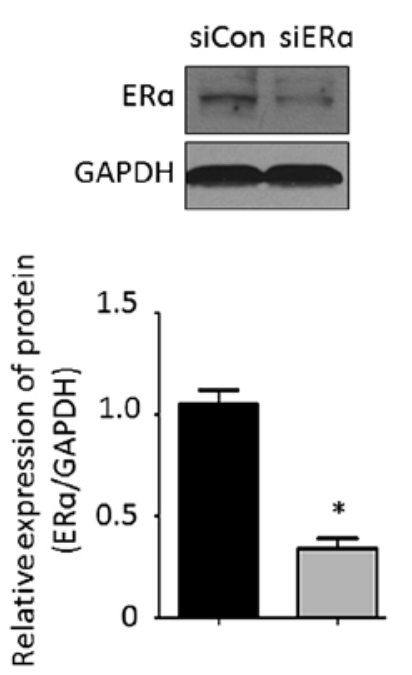

$\mathrm{B}$
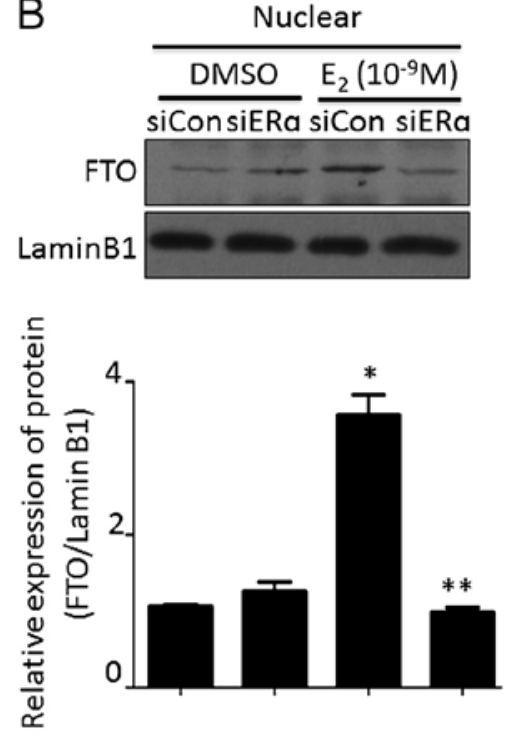

C

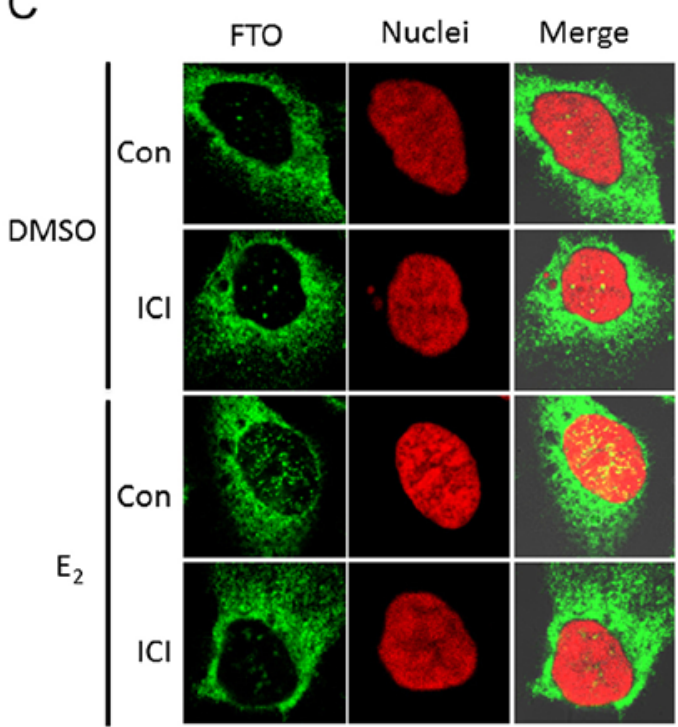

Figure 4. ER $\alpha$ knockdown is required for estrogen-induced FTO nuclear localization. (A) Transfection efficiency of siER $\alpha$ was determined by western blotting. (B) The effect of knockdown of ER $\alpha$ on FTO nuclear localization after estrogen stimulation. (C) Immunocytochemical assay was used to determine the decrease in FTO nuclear accumulation resulting from ICI $(1 \mu \mathrm{M})$ treatment.

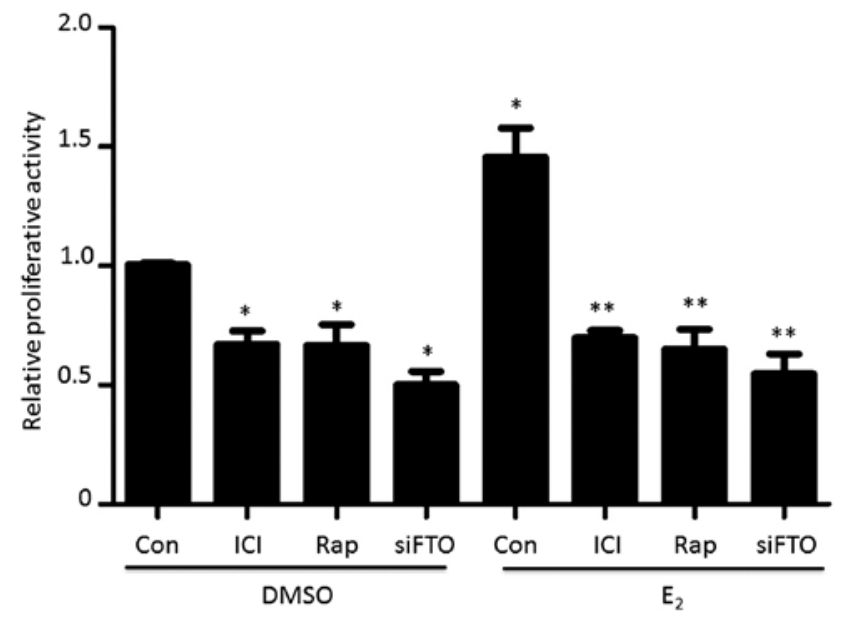

Figure 5. Estrogen-induced FTO nuclear localization promotes endometrial cancer cell proliferation. After treatment with ICI $(1 \mu \mathrm{M})$, rapamycin (Rap) $(100 \mathrm{nM})$ and siFTO, Ishikawa cells were treated with $10^{-9} \mathrm{M}$ estrogen or DMSO for another $48 \mathrm{~h}$. MTT assay was used to evaluate the cell proliferative activity. ${ }^{*} \mathrm{P}<0.05$, compared with the control. ${ }^{* *} \mathrm{P}<0.01$, compared with the control plus $10^{-9} \mathrm{M}$ estrogen stimulation.

ER $\alpha$ is required for estrogen-induced FTO nuclear localization. ER $\alpha$ plays an important role in estrogen-mediated bio-functions. Therefore, we investigated the effect of ER $\alpha$ on estrogen-induced FTO nuclear localization. As shown in Fig. 4A, acute transfection of siER $\alpha$ resulted in a marked decrease in ER $\alpha$ protein, which had no effect on FTO nuclear expression. However, estrogen-induced FTO expression in the nucleus was blocked by deletion of ER $\alpha$ (Fig. 4B). Immunocytochemistry assay demonstrated that estrogen stimulation accumulated FTO protein in the nucleus, however, the increased FTO protein dot in the nucleus was abolished by $1 \mu \mathrm{M}$ fulvestrant (ICI 182,780; purchased from Sigma-Aldrich) treatment, a selective estrogen receptor antagonist (Fig. 4C).
These data suggested that $\mathrm{ER} \alpha$ is required for estrogeninduced FTO nuclear localization.

Estrogen-induced FTO nuclear localization promotes endometrial cancer cell proliferation. To understand FTO nuclear localization in endometrial cancer proliferation, we carried out an MTT assay. As shown in Fig. 5, pretreatment with ICI, to block the ER $\alpha$ signaling pathway, attenuated Ishikawa cell proliferative activity. Moreover, ICI treatment also blocked estrogen-induced cell growth. Similarly, blocking the mTOR signaling pathway with rapamycin also abolished the estrogen-stimulated cell proliferation. Targeting FTO by siFTO directly resulted in decreases proliferative activity, whereas estrogen treatment could not rescue the cellular growth.

\section{Discussion}

In the present study, we found that the fat mass and obesityassociated (FTO) gene was overexpressed in endometrial carcinoma tissues and estrogen induced FTO nuclear localization, which facilitated endometrial cancer proliferation through the mTOR signaling pathway.

Most endometrial adenocarcinomas are characterized by positive nuclear estrogen receptor (ER) expression and responsiveness to hormone stimulation. Increasing evidence indicates that prolonged estrogen exposure is associated with initiation of type 1 endometrioid cancers (29-31). Estrogen exposure was found to result in an overall physiological response within several hours by a genomic mechanism which depends on estrogen binding to nuclear ER resulting in mRNA transcription and protein synthesis of target genes. Obesity is a well-established risk factor for endometrial cancer since obesity in post-menopausal women has been shown to increase circulating estrogen levels by upregulating the expression of aromatase and enhancing aromatization of androstenedione in 
adipose tissue. We documented that the FTO gene is involved in estrogen-driven endometrial cancer development. However, the detail molecular mechanism remains to be clarified.

An investigation with a larger sample size confirmed that the obesity-associated polymorphism FTO rs9939609 is strongly associated with endometrial cancer risk in non-Hispanic white women (13). In the present study, we re-evaluated the expression of FTO in endometrial cancer and the association with prognosis. The higher expression in endometrial carcinoma tissues was observed compared with the normal endometrial tissues. In our previous study, we found that there is no association between FTO expression and age, stage, grade, invasion and lymph node metastasis (7). Notably, we found that higher FTO expression was related with poor prognosis and early relapse. These data imply that FTO plays an important role in endometrial cancer development. It was observed that estrogen stimulation enhanced FTO protein accumulation in endometrial cancer cell nuclei. Yet, limited information is available concerning its mechanism and its effect on cellular growth. Consistent with our previous study (7), estrogen upregulated FTO protein in a dose- and time-dependent manner (Fig. 2A and B). We further isolated the nuclear and cytosolic proteins and found that in fact estrogen did not increase the FTO protein level in the cytoplasm, whereas elevated FTO protein by estrogen in the nucleus was observed. It is known that protein is synthesized in the cytoplasm, but we did not detect any alteration in FTO protein in the cytoplasm after estrogen treatment, which suggests that estrogen induces FTO to transfer into the nucleaus.

A previous study demonstrated that mTOR signaling is involved in estrogen-driven endometrial cancer development (32). In the present study, we provided evidence that mTOR signaling controls estrogen-induced FTO protein nuclear localization. As shown in Fig. 3A and B, estrogen activated mTOR signaling and this activation was inhibited by rapamycin, an mTOR specific inhibitor. Most importantly, rapamycin blocked the accumulation of FTO in the nucleus with or without estrogen stimulation (Fig. 3C and D). These data suggest that activation of mTOR signaling is necessary for FTO nuclear localization.

Given that ER $\alpha$ mediated estrogen-induced multiple functions, we further investigated the role of ER $\alpha$ in FTO nuclear localization. In the present study, we found that knockdown of ER $\alpha$ had a slight effect on FTO protein nuclear localization. However, deletion of ER $\alpha$ blocked estrogen-induced FTO nuclear accumulation. We considered that ER $\alpha$ has no effect on FTO expression, but estrogen-mediated function is $E R \alpha$-dependent. Therefore, depletion of ER $\alpha$ resulted in the inability of estrogen to bind with ER $\alpha$, in turn leading to estrogen-driven FTO nuclear accumulation failure. These data imply that ER $\alpha$ is required for FTO nuclear localization. Although we gathered significant data in the present study, to the best of my knowledge, ER $\alpha$ was also located in the nucleus and served as a transcription factor. Yet, we still raised the question of how ER $\alpha$ mediates FTO nuclear accumulation. We hypothesized that ER $\alpha$ may recruit some molecules and construct a protein complex, which in turn helps FTO to transfer into the nucleus. Various proteins have different functions when they present in different cellular localizations, such as caveolin. In the present study, we found that FTO nuclear localization promoted cell proliferation, whereas blocking the nuclear accumulation by ICI or Rap pretreatment, even by direct deletion of FTO by siRNA, resulted in attenuated proliferative activity. The decreased proliferation could not be rescued by estrogen stimulation.

In conclusion, the present study suggests that overexpression of FTO in endometrial carcinoma may be a poor prognostic marker. Importantly, FTO nuclear accumulation may be an essential step for estrogen-driven endometrial tumor formation and progression. Our findings may provide new insight into the mechanisms underlying E2-induced proliferation. Additionally, the present study further supports the possibility of using FTO as a target for the treatment of endometrial cancer.

\section{Acknowledgements}

The present study was supported by grants from the National Natural Science Foundation of China (NSFC no. 81372795).

\section{References}

1. Chan S: A review of selective estrogen receptor modulators in the treatment of breast and endometrial cancer. Semin Oncol 29 (Suppl 11): 129-133, 2002.

2. Ito K, Utsunomiya H, Yaegashi $\mathrm{N}$ and Sasano H: Biological roles of estrogen and progesterone in human endometrial carcinoma - new developments in potential endocrine therapy for endometrial cancer. Endocr J 54: 667-679, 2007.

3. Bokhman JV: Two pathogenetic types of endometrial carcinoma. Gynecol Oncol 15: 10-17, 1983

4. Bochkareva NV, Kolomiets LA, Kondakova IV, Stukanov SL, Starova AB and Agarkova LA: Enzymes of estrogen metabolism in endometrial cancer. Bull Exp Biol Med 141: 240-242, 2006.

5. Chen Y, Huang Q, Chen Q, Lin Y, Sun X, Zhang H, Zhu M and Dong S: The inflammation and estrogen metabolism impacts of polychlorinated biphenyls on endometrial cancer cells. Toxicol In Vitro 29: 308-313, 2015.

6. Williams-Brown MY, Salih SM, Xu X, Veenstra TD, Saeed M, Theiler SK, Diaz-Arrastia CR and Salama SA: The effect of tamoxifen and raloxifene on estrogen metabolism and endometrial cancer risk. J Steroid Biochem Mol Biol 126: 78-86, 2011.

7. Zhang Z, Zhou D, Lai Y, Liu Y, Tao X, Wang Q, Zhao G, Gu H, Liao H, Zhu Y, et al: Estrogen induces endometrial cancer cell proliferation and invasion by regulating the fat mass and obesityassociated gene via PI3K/AKT and MAPK signaling pathways. Cancer Lett 319: 89-97, 2012.

8. da Cunha PA, de Carlos Back LK, Sereia AF, Kubelka C, Ribeiro MC, Fernandes BL and de Souza IR: Interaction between obesity-related genes, $F T O$ and $M C 4 R$, associated to an increase of breast cancer risk. Mol Biol Rep 40: 6657-6664, 2013.

9. Gaudet MM, Yang HP, Bosquet JG, Healey CS, Ahmed S, Dunning AM, Easton DF, Spurdle AB, Ferguson K, O'Mara T et al: No association between FTO or HHEX and endometrial cancer risk. Cancer Epidemiol Biomarkers Prev 19: 2106-2109, 2010.

10. Hernández-Caballero ME and Sierra-Ramírez JA: Single nucleotide polymorphisms of the FTO gene and cancer risk: An overview. Mol Biol Rep 42: 699-704, 2015.

11. Kaklamani V, Yi N, Sadim M, Siziopikou K, Zhang K, Xu Y, Tofilon S, Agarwal S, Pasche B and Mantzoros C: The role of the fat mass and obesity associated gene $(F T O)$ in breast cancer risk. BMC Med Genet 12: 52, 2011.

12. Lewis SJ, Murad A, Chen L, Davey Smith G, Donovan J, Palmer T, Hamdy F, Neal D, Lane JA, Davis M, et al: Associations between an obesity related genetic variant (FTO rs9939609) and prostate cancer risk. PLoS One 5: e13485, 2010.

13. Lurie G, Gaudet MM, Spurdle AB, Carney ME, Wilkens LR, Yang HP, Weiss NS, Webb PM, Thompson PJ, Terada K, et al; Australian National Endometrial Cancer Study Group; Epidemiology of Endometrial Cancer Consortium (E2C2): The obesity-associated polymorphisms FTO rs9939609 and MC4R rs17782313 and endometrial cancer risk in non-Hispanic white women. PLoS One 6: e16756, 2011. 
14. Fischer J, Koch L, Emmerling C, Vierkotten J, Peters T, Brüning JC and Rüther U: Inactivation of the Fto gene protects from obesity. Nature 458: 894-898, 2009.

15. Wong M: A critical review of mTOR inhibitors and epilepsy: From basic science to clinical trials. Expert Rev Neurother 13: 657-669, 2013

16. Xu J and Tian D: Hematologic toxicities associated with mTOR inhibitors temsirolimus and everolimus in cancer patients: A systematic review and meta-analysis. Curr Med Res Opin 30: 67-74, 2014.

17. Atefi M, von Euw E, Attar N, Ng C, Chu C, Guo D, Nazarian R, Chmielowski B, Glaspy JA, Comin-Anduix B, et al: Reversing melanoma cross-resistance to BRAF and MEK inhibitors by co-targeting the AKT/mTOR pathway. PLoS One 6: e28973, 2011.

18. Jazirehi AR, Wenn PB and Damavand M: Therapeutic implications of targeting the PI3Kinase/AKT/mTOR signaling module in melanoma therapy. Am J Cancer Res 2: 178-191, 2012.

19. Pópulo H, Soares P and Lopes JM: Insights into melanoma: Targeting the mTOR pathway for therapeutics. Expert Opin Ther Targets 16: 689-705, 2012.

20. Bae SY, Kim GD, Jeon JE, Shin J and Lee SK: Anti-proliferative effect of (19Z)-halichondramide, a novel marine macrolide isolated from the sponge Chondrosia corticata, is associated with G2/M cell cycle arrest and suppression of mTOR signaling in human lung cancer cells. Toxicol In Vitro 27: 694-699, 2013.

21. Dong LX, Sun LL, Zhang X, Pan L, Lian LJ, Chen Z and Zhong DS: Negative regulation of mTOR activity by LKB1-AMPK signaling in non-small cell lung cancer cells. Acta Pharmacol Sin 34: 314-318, 2013.

22. Papadimitrakopoulou V: Development of PI3K/AKT/mTOR pathway inhibitors and their application in personalized therapy for non-small-cell lung cancer. J Thorac Oncol 7: 1315-1326, 2012.

23. Bradford LS, Rauh-Hain A, Clark RM, Groeneweg JW, Zhang L, Borger D, Zukerberg LR, Growdon WB, Foster R and Rueda BR: Assessing the efficacy of targeting the phosphatidylinositol 3-kinase/AKT/mTOR signaling pathway in endometrial cancer. Gynecol Oncol 133: 346-352, 2014.
24. Chen J, Zhao KN, Li R, Shao R and Chen C: Activation of PI3K/ $\mathrm{Akt} / \mathrm{mTOR}$ pathway and dual inhibitors of PI3K and mTOR in endometrial cancer. Curr Med Chem 21: 3070-3080, 2014.

25. Cheng H, Liu P, Zhang F, Xu E, Symonds L, Ohlson CE, Bronson RT, Maira SM, Di Tomaso E, Li J, et al: A genetic mouse model of invasive endometrial cancer driven by concurrent loss of Pten and Lkb1 Is highly responsive to mTOR inhibition. Cancer Res 74: 15-23, 2014

26. Myers AP: New strategies in endometrial cancer: Targeting the $\mathrm{PI} 3 \mathrm{~K} / \mathrm{mTOR}$ pathway - the devil is in the details. Clin Cancer Res 19: 5264-5274, 2013.

27. Pavlidou A and Vlahos NF: Molecular alterations of PI3K/Akt/ mTOR pathway: A therapeutic target in endometrial cancer. ScientificWorldJournal 2014: 709736, 2014.

28. Zhang Z, Dong L, Sui L, Yang Y, Liu X, Yu Y, Zhu Y and Feng Y: Metformin reverses progestin resistance in endometrial cancer cells by downregulating GloI expression. Int J Gynecol Cancer 21: 213-221, 2011.

29. Chao A, Lin CY, Tsai CL, Hsueh S, Lin YY, Lin CT, Chou HH, Wang TH, Lai $\mathrm{CH}$ and Wang HS: Estrogen stimulates the proliferation of human endometrial cancer cells by stabilizing nucleophosmin/B23 (NPM/B23). J Mol Med Berl 91: 249-259, 2013.

30. Thorne AM, Jackson TA, Willis VC and Bradford AP: Protein kinase $\mathrm{C} \alpha$ modulates estrogen-receptor-dependent transcription and proliferation in endometrial cancer cells. Obstet Gynecol Int 2013: 537479, 2013.

31. Zhou C, Steplowski TA, Dickens HK, Malloy KM, Gehrig PA, Boggess JF and Bae-Jump VL: Estrogen induction of telomerase activity through regulation of the mitogen-activated protein kinase (MAPK) dependent pathway in human endometrial cancer cells. PLoS One 8: e55730, 2013.

32. Hou X, Zhao M, Wang T and Zhang G: Upregulation of estrogen receptor mediates migration, invasion and proliferation of endometrial carcinoma cells by regulating the $\mathrm{PI} 3 \mathrm{~K} / \mathrm{AKT} / \mathrm{mTOR}$ pathway. Oncol Rep 31: 1175-1182, 2014. 